\title{
MODELS AND ALGORITHMS FOR ESTIMATION AND MINIMIZATION OF THE RISKS ASSOCIATED WITH DREDGING
}

\author{
D. Mamunts, S. Sokolov, A. Nyrkov, S. Chernyi, M. Bukhurmetov, V. Kuznetsov \\ Admiral Makarov State University of Maritime and Inland Shipping \\ 198035, Russia, Saint-Petersburg, Dvinskayast., 5/7 \\ E-mail: sergiiblack@gmail.com
}

\begin{abstract}
There are a lot of models and algorithms to minimize risks during dredging operations and they are not without drawbacks. The paper describes the authors' approach to solving this problem. Mathematical models are proposed and on their basis software is developed. Methods of the risk theory are used to minimize the risks. In this paper a consequence of influence refers to the deviation from the goal expressed in the expected results and the deviation of certain criterion factors. In this case, we mean any measure of quality. In its turn, risk factors reduce criterion factors. These factors are divided into categories - general transportation risks and risks of transporting ground. In these categories, one may derive the following risks - incidents at transport resulting from the impact of a set of random factors including the human one. For risk analysis and management, in addition to identifying critical chains of risk situations, the stochastic model for evaluating the chains is set forth. In order to implement this algorithm, the mathematical package Maple is used, which allows for conducting the required calculations with a software package including the Graph Theory. The paper presents fragments of the code listing.
\end{abstract}

Keywords: dredging operations, risks assessment, risks minimization

\section{Introduction}

Dredging is a kind of underwater operations at the bottom of a water body in order to create new waterways or to deepen the existing ones, to construct piers, bridges and other hydraulic and water facilities as well as to expand and increase the depth of water bodies. Dredging is divided into capital and operational. Capital dredging is performed in order to enhance the quality of navigational conditions as well as to construct hydro technical facilities (Vieira et al., 2015). Operational dredging is undertaken in order to clean water facilities. Dredging is the main method of ensuring the specified size for ships' traffic and ameliorating shipping. Dredging is carried out by means of dredgers, extracting and dumping the ground out of the riverbed areas difficult for navigation (Chernyi, 2016a). Dredging is an inherently complex technical process that depends on a large number of random factors. Dredging involves great technological, technological, economic and environmental risks (Spearman, 2015). The process of assessing these risks (Nyrkov et al., 2015) described in this paper is a tool aimed at helping in identifying hazards and formulating the problem in such a way as to reduce the risk to an acceptable level. Risk assessment consists of a frequency analysis, consequence analysis and their aggregate (Malioutov et al., 2016). Major tasks for the risk assessment phase include determining the frequency of occurrence of initiating and of all unwanted events; assessment of the impact of adverse events and compilation of risk assessments.

When summarizing the risks assessments it is recommended that uncertainties and the accuracy of the results be analyzed. In most cases, the source of uncertainty in the case of dredging will be incomplete data on the performance technology, reliability and accuracy of equipment operation as well as human error. To interpret the results of the risk assessment correctly, it is recommended to be identified, evaluated and submitted as the results.

\section{Methods Description}

In practice qualitative or quantitative risk assessment was used (Chernyi, 2016b). The qualitative risk analysis identifies the sources and causes of risk, stages and operations in the course of which there is a risk (presence of potential risk areas, identification of associated risk activities, finding out practical benefits and negative effects of the identified risks). The quantitative analysis also provides a numeric value for both individual risks and for the entire facility it is also to calculate the potential damage and to develop a system of preventive measures aimed at minimizing the occurrence of conditions for risk 
situations (Rood, 2009). In analyzing risks, the risk matrix and generic risk assessment algorithm were used.

The matrix of risk assessment with dredging based on the principles used in the risk assessment theory. The risk matrix is shown in Figure 1. To assess risks a team of experts evaluate the level of their weight for each identified risk (the impact on the project or its outcome / level of the incident, accident or situation close to it) and its probability of occurrence (the number of times the risk may occur or the probability of the risk). As a result, the level of risk (from very high to very low) is estimated for each element (Upadhya et al., 2016). Once the risk level is determined by the severity (x-axis of the graph) and probability (y-axis of the graph), risk index scores are selected for each hazard. The risk index is a measure of the magnitude of probable losses (in points); it makes it possible to judge the degree of exposure and risk level.

$R=S q \times P q$,

where: $R$ is a risk index;

$S_{q}$ is the risk cost;

$P_{q}$ is the probability of risk occurrence.

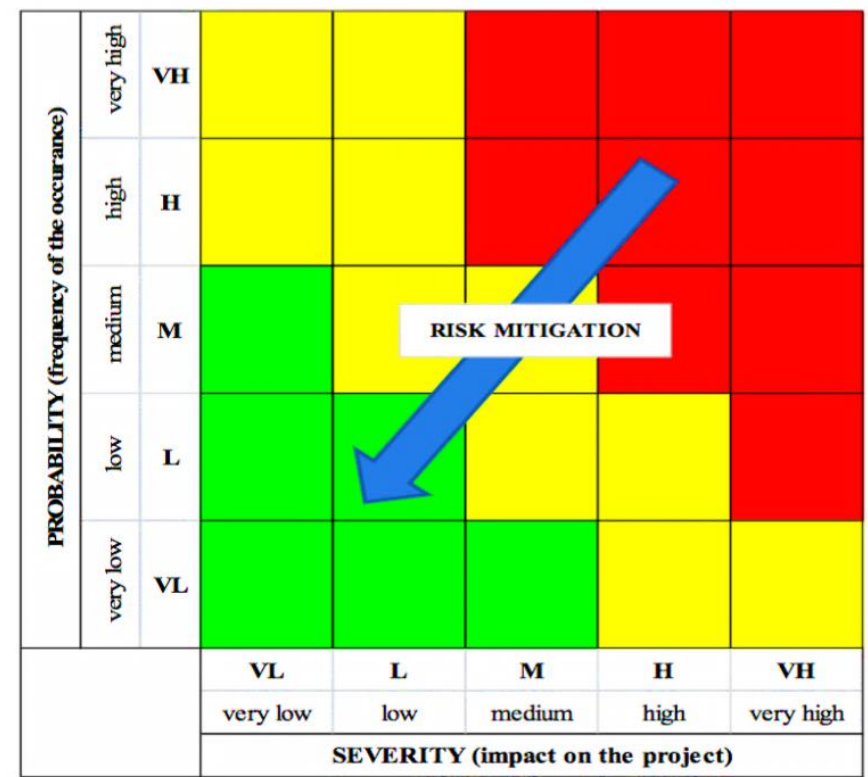

Figure 1. Risk assessment matrix

There are many models and algorithms to analyse and minimize risks: economical, technical and informational. In this paper we used traditional a risk definition (Chen and Cheng, 2014). Risk is a consequence of the impact of uncertainty on the achievement of goals. For the purposes of the paper, a consequence of influence refers to as the deviation from the goal expressed in the expected result and rejection of certain criterion factors. In this case, we can treat it as any quality indicator (volume of soil, width / depth of the slots in a certain area, cost of dredging). In its turn, to reduce risk factors there were used criterion factors. These factors are divided into categories: general transportation risks and the risks of ground transportation. In these categories, risks are divided into incidents in transport caused by the influence of a set of random factors including a human one.

To analyze and manage risks, in addition to identifying the critical chains of risk situations, a stochastic model for determining the chains was proposed. Taking into account a large number of risk situations determined empirically, the matrix dimension to the adjacency of this graph was used to allow for the application of the exhaustive algorithm (Ruffio et al., 2012). To implement this algorithm, the mathematical package Maple (Tuan, 2013) is used in which it is possible to carry out the required calculations applying software package GraphTheory. The paper presents fragments of the code listing.

Quantification of the effect of the $i$-th factor for risk $Z_{i}$ is calculated with a mean value of the loss criterion parameter in the implementation of this risk: $\mathrm{Z}_{\mathrm{i}}=\mathrm{p}_{\mathrm{i}} \Delta \mathrm{Zi}$, where $p_{i}$ is the probability of the $i$-th risk factor, and $\Delta Z_{i}$ is an absolute loss in its implementation. Depending on the occurrence of one of $m_{i}$ of 
non-coupled events $A_{i j}$ with probability $p_{i j}$, if the $i$-th risk can appear, the quantification of the level of this risk will be assessed from the ratio:

$Z_{i}=\sum_{j=1}^{m_{i}} p_{i j} \times \Delta Z_{i j}$

where $\boldsymbol{p}_{i j}=\mathbf{P}\left(\left\{\boldsymbol{Z}_{\boldsymbol{i}}=\Delta \boldsymbol{Z}_{i j}\right\} / \boldsymbol{A}_{i j}\right)$ is the probability that the occurrence of event $A_{i j}$ results in the reduction of the criterion factor for $\Delta Z_{i j}$.

In general, the evaluation of the criterion index reduction can be obtained as the sum of the average potential losses due to the existence of each of the $n$ risks:

$Z=\sum_{i=1}^{n} Z_{i}=\sum_{i=1}^{n} \sum_{j=1}^{m_{i}} p_{i j} \times \Delta Z_{i j}$.

The simultaneous occurrence of risky situations is unlikely. It should also be taken into account that the occurrence of a risk situation can determine and have a direct impact on the other. As a result, one may experience the chains of risks associated with each other. By constructing an acyclic digraph for risk situations (Cutroneo et al., 2015), it is proposed to determine the only situation among all the risk ones that may lead to the maximum possible loss of the criterion factors. Let be $G=(V, E)$ an acyclic digraph, where $V=\{1,2, \ldots, n\}$ is a set of graph nodes, $E=\{(i, j)\}$ is a set of arcs. Passing from the node $i$ to the node $j$, the arc $(i, j)$ is included into the graph $\mathrm{G}$ if the risk situation $j$ can follow the risk situation $i$. In implementing the risk situation $i$, the arc length or its weight coefficient $Z_{i}$ is set to correspond to a possible value loss of the criterion parameter. Acyclic graph nodes may be numbered in such a way that the equation $i>j$ will be suitable for all the $\operatorname{arcs}(i, j)$. The node with the number 1 is associated with the criterion factor, the rest of the nodes - with possible risk situations. To find the most critical chain of risk situations, an arbitrary chain will be represented as a vector:

$X=\left\{x_{i j} \mid(i, j) \in G\right\}$.

With this $x_{i j}=1$, if the $\operatorname{arc}(i, j)$ belongs to the chain under review, otherwise $x_{i j}=0$. Vector $X=$ $\left\{x_{i j} \mid(i, j) \in G\right\}$ describes the chain, passing through the nodes of the graph $G$, if the following conditions are satisfied:

$$
\begin{aligned}
& \sum_{(i, j) \in G} x_{i j}-\sum_{(j, i) \in G} x_{j i}=0, \text { if } i \in\{2,3, \ldots, n-1\} ; \sum_{(i, j) \in G} x_{i j}-\sum_{(j, i) \in G} x_{j i}=0, \text { if } i \in\{2,3, \ldots, n-1\} ; \\
& \sum_{(1, j) \in G} x_{1 j}-\sum_{(j, 1) \in G} x_{j 1}=-1 \sum_{(1, j) \in G} x_{1 j}-\sum_{(j, 1) \in G} x_{j 1}=-1 ; \\
& \sum_{(n, j) \in G} x_{n j}-\sum_{(j, n) \in G} x_{j n}=1 \sum_{(n, j) \in G} x_{n j}-\sum_{(j, n) \in G} x_{j n}=1 .
\end{aligned}
$$

The possible loss of the criterion factor for risky situation $X$ may be assessed by the ratio

$Z(X)=\sum_{(i, j) \in G} x_{i j} Z_{i j}$

where $Z_{i j}=Z_{i}$, if the $\operatorname{arc}(i, j)$ is a part of the graph $\mathrm{G}$, otherwise $Z_{i j}=0$.

In order to assess the critical chain of risky situations the following model is proposed:

$$
\left\{\begin{array}{l}
\max \{Z(\mathbf{X})\}, \\
\sum_{(1, j) \in G} x_{1 j}-\sum_{(j, 1) \in G} x_{j 1}=-1, \\
\sum_{(i, j) \in G} x_{i j}-\sum_{(j, i) \in G} x_{j i}=0, i \in\{2,3, \ldots, n-1\}, \\
\sum_{(n, j) \in G} x_{n j}-\sum_{(j, n) \in G} x_{j n}=1, \\
x_{i j} \in\{0,1\}, \forall(i, j) \in G .
\end{array}\right.
$$

For larger dimensions of the adjacency matrix of graph $\mathrm{G}$, there are algorithms making possible to find the maximum length of the chain quickly and efficiently (Walther et al., 2016). By using the 
"greedy" algorithm, such as Dijkstra's algorithm, and by altering the sign for all the weights and taking into account the acyclic graph $G$, it is plausible to construct an algorithm for finding the critical chain, the time complexity of which implementation as compared with the total number of nodes and arcs is linear. For the acyclic directed graph, one can get such a linearly ordered sequence of nodes that the initial node of any arc of this graph is located in it before the final node of the arc (Russell, 2014). This means that one can represent all the nodes of the graph as $v_{l}, v_{2}, \ldots, v_{n}$, and the node $v_{i}$ precedes to the node $v_{j}(i<j)$ if the graph has an arc $\left(v_{i}, v_{j}\right)$. This can significantly reduce the time complexity of the algorithm for identification of the critical chain of risk situations (Khoshgoftaar, 2003; Chernyi, 2016c). Moreover, it is possible to realize an algorithm that identifies not one but all critical chains.

To account for the loss of the stochastic criterion index, $Z_{i j}(7)$ will be considered as a random variable having a probability density function for a continuous random variable $p_{Z_{i j}}(z)$ or subordinate in the law of probability distribution of a discrete random variable $\left\{Z_{i j}^{k}->p_{i j}^{k}=P\left(Z_{i j}=Z_{i j}^{k}\right)\right\}, k=1 . . n_{i}$ $\left\{\mathrm{Z}_{\mathrm{ij}}^{\mathrm{k}} \rightarrow \mathrm{p}_{\mathrm{ij}}^{\mathrm{k}}=\mathrm{P}\left(\mathrm{Z}_{\mathrm{ij}}=\mathrm{Z}_{\mathrm{ij}}^{\mathrm{k}}\right)\right\}, k=1, \ldots, n_{i}$. Let add another restriction to the limitations of the model (8)

$\mathbf{P}\left\{\sum_{(i, j) \in G} x_{i j} Z_{i j} \geq Z_{K P}\right\} \geq 1-\alpha \mathrm{P}\left\{\sum_{(\mathrm{i}, \mathrm{j}) \in \mathrm{G}} \mathrm{x}_{\mathrm{ij}} \mathrm{Z}_{\mathrm{ij}} \geq \mathrm{Z}_{\mathrm{KP}}\right\} \geq 1-\alpha$.

where $\alpha$ is a predetermined significance level.

The stochastic model for the critical chain is estimated as

$$
\left\{\begin{array} { l } 
{ \operatorname { m a x } \{ Z _ { K P } \} , } \\
{ \mathbf { P } \{ \sum _ { ( i , j ) \in G } x _ { i j } Z _ { i j } \geq Z _ { K P } \} \geq 1 - \alpha } \\
{ \sum _ { ( 1 , j ) \in G } x _ { 1 j } - \sum _ { ( j , 1 ) \in G } x _ { j 1 } = - 1 , } \\
{ \sum _ { ( i , j ) \in G } x _ { i j } - \sum _ { ( j , i ) \in G } x _ { j i } = 0 , i \in \{ 2 , 3 , \ldots , n - 1 \} , } \\
{ \sum _ { ( n , j ) \in G } x _ { n j } - \sum _ { ( j , n ) \in G } x _ { j n } = 1 , } \\
{ x _ { i j } \in \{ 0 , 1 \} , \forall ( i , j ) \in G . }
\end{array} \left\{\begin{array}{c}
\max \left\{Z_{K P}\right\}, \\
P\left\{\sum_{(i, j) \in G} x_{i j} Z_{i j} \geq Z_{K P}\right\} \geq 1-\alpha \\
\sum_{(1, j) \in G} x_{1 j}-\sum_{(j, 1) \in G} x_{j 1}=-1, \\
\sum_{(i, j) \in G} x_{i j}-\sum_{(j, i) \in G} x_{j i}=0, i \in\{2,3, \ldots, n-1\}, \\
\sum_{(n, j) \in G} x_{n j}-\sum_{(j, n) \in G} x_{j n}=1, \\
x_{i j} \in\{0,1\}, \forall(i, j) \in G
\end{array}\right.\right.
$$

According to the analysis of the situational management risk in the chain of critical events, stochastic models can be applied. The model (10) displays a situational approach to identify the significance level of the model. Such a model can be formulated as following:

$\max P\left\{\sum_{(i, j) \in G} x_{i j} Z_{i j} \geq Z_{K P}\right\}$,

with limitations

$$
\left\{\begin{array}{c}
\sum_{(1, j) \in G} x_{1 j}-\sum_{(j, 1) \in G} x_{j 1}=-1, \\
\sum_{(i, j) \in G} x_{i j}-\sum_{(j . i) \in G} x_{j i}=0, i \in\{2,3, \ldots, n-1\}, \\
\sum_{(n, j)} x_{n j}-\sum_{(j, n) \in G} x_{j n}=1, \\
x_{i j} \in\{0,1\}, \forall(i, j) \in G
\end{array}\right.
$$

These models and algorithms may be successfully modified and used in other industries.

It is possible to offer modification of the "method of random search" as the basis of algorithms to determine appropriate models for chains (10) and (11) (Tuan, 2013).

The integrated algorithm for the model (10) is constructed as follows.

1. Assign $k$ value 1 .

2. Generate the risky chain $\left\{x_{i j}^{k}\right\}$.

3. Increase $k$ by 1 . Repeat step 2 if $k$ is not greater than $\mathrm{N}$. 
4. Put $\mathrm{M}$, equal to the whole $\operatorname{part}(1-\alpha) N$.

5. The maximum value $Z_{K P}$ is set equal to the length of the M-th largest chain satisfying the constraint (9).

An enlarged model algorithm (11) is developed as follows.

1. For a given number of generation series of risk situations - $\mathrm{N}$ sequences, set the maximum value $Z_{K P}$.

2. Ask M - the number of generated sequences in the series. This value must be greater than five times the number of arcs in the graph G. Put $k$, equal to 1.

3. Generate a series of $M$ risky chains $\left\{\left\{x_{i j}^{1}\right\}, \ldots,\left\{x_{i j}^{M}\right\}\right\}$,

4. as the chain $\left\{x_{i j}^{k}\right\}$ to take the length of which is most likely to exceed the maximum value $Z_{K P}$ (Park, 2015).

5. Increase $k$ by 1 . Repeat steps 3 and 4 , if $k$ is not greater than $\mathrm{N}$.

6 . From these chains in paragraph 4 select one that has a maximum repetition rate.

\section{Software for Identifying the Critical Chain of Risk Situations}

Algorithms for identifying a critical chain of risk situations implemented in the program Maple environment. It is assumed that the risk of exposure to the lowering of criterion indicators is investigated (Shi and $\mathrm{Ji}, 2014$ ). The input data for each risk situation are relative value decline criterion for the implementation of this risk. For example, the value 0.012 means a reduction criterion index by $1.2 \%$. For each risk reduction criterion and in addition to the quantities specified, peer review of probability presents two-dimensional array of $\mathrm{K}_{\mathrm{i}}$ values.

Procedure ranKi:=proc(tab1::list,tab2::list, n::integer, out Y::evaln) creates an array

Ki_r4 $\left\{\begin{array}{c}\sum_{(1, j) \in G} x_{1 j}-\sum_{(j, 1) \in G} x_{j 1}=-1, \\ \sum_{(i, j) \in G} x_{i j}-\sum_{(j . i) \in G} x_{j i}=0, i \in\{2,3, \ldots, n-1\}, \\ \sum_{(n, j)} x_{n j}-\sum_{(j, n) \in G} x_{j n}=1, \\ x_{i j} \in\{0,1\}, \forall(i, j) \in G\end{array}\right.$

in which the risk of value is represented by the corresponding stochastic variable. Interrelation of risk situations is given a directed graph with the vertices $V_{i} \operatorname{arcs} E$.

It finds and prints the string having the greatest impact on criterion factors. For models (10) and (11) of each arc of the graph is generated by the risk Graph weight corresponding to the effect of the risk situation on criterion factors. The number of tests is specified in the variable nIteration. Operations in model (10) are conducted to find the critical chain procedure.

\section{Approbation Results}

Models and algorithms were tested and approbated for the environmental impact assessment of dredging at the River Svir at 2015 year. The main task at the stage of environmental impact assessment is an enlarged assessment of the main parameters of the planned economic activity and identification of the main risks in the event of its possible implementation. These materials are the key instrument for identifying the main risks of the impact on the environment and people living in the vicinity of the planned construction.

Research includes the following components of assessment: assessment of water resources, assessment of atmospheric air and assessment of soil. The part of risk assessment results is shown in Table 1.

These results were formed automatically by using a program code based on the mentioned method and algorithm. Before using this program one needs to develop a criterion analysis for each part and include weight of point to make result easier to understand. 
Table 1. Risk assessment results

\begin{tabular}{|l|l|l|l|l|l|}
\hline \multicolumn{1}{|c|}{ Activity Description } & \multicolumn{1}{|c|}{ Potential Consequences } & Consequence & Likelihood & Rating & Control Strategy \\
\hline $\begin{array}{l}\text { Impact on seagrass and } \\
\text { mangrove via changes } \\
\text { to the hydrodynamic } \\
\text { regime of a river }\end{array}$ & $\begin{array}{l}\text { Potential changes in } \\
\text { community structure, loss of } \\
\text { resources for protected } \\
\text { species and fisheries }\end{array}$ & 4 & 3 & M & Mydrodynamic \\
modelling \\
$\begin{array}{l}\text { Potential of } \\
\text { contaminants in } \\
\text { dredging material }\end{array}$ & $\begin{array}{l}\text { Impact to human health, land } \\
\text { use, release of contaminants } \\
\text { into water, potential financial } \\
\text { loss }\end{array}$ & 3 & 3 & $\begin{array}{l}\text { Undertake } \\
\text { environmental } \\
\text { investigations before } \\
\text { commencing dredging }\end{array}$ \\
\hline $\begin{array}{l}\text { Oil spills from } \\
\text { construction vehicles }\end{array}$ & $\begin{array}{l}\text { Potential environmental } \\
\text { impact }\end{array}$ & 1 & 4 & L & $\begin{array}{l}\text { Maintain vehicle } \\
\text { routine inspections }\end{array}$ \\
\hline
\end{tabular}

\section{Conclusions}

The model of assessment and the algorithm to minimize the risks arising from the dredging which are given in the paper are based on the classical approach, which initially included the method of expert estimation. Then, the paper provides the algorithm for constructing the chains (including finding the most critical of them), which uses a "greedy" algorithm like Dijkstra's algorithm, for the sensitive analysis of possible chains in risk situations. The difference is that the sign of all the weights in the acyclic graph of the balance is reversed. A distinctive feature of the algorithm is a linear dependence of the algorithm time complexity on the number of vertices and edges.

After the construction of chains of risk events the second most important way in the assessment of risk situations is the stochastic model for determining the chains with the probability that the criterion index loss will be extreme from the predetermined level of significance $\alpha$. The software based on the model and the algorithm show the real cases of using the developed action sequences and patterns, from which it is clear that the use of the proposed model and algorithm may be extended to other areas (not necessarily adjacent).

As an extension of the study and in order to assess the impacts of threats at the level of safety on water transport modes as well as water transport infrastructure safety in general, in future the authors are planning to conduct risk assessment in the related fields and to form a combination of risk situations examining them under the condition of mutual influence.

\section{References}

1. Chen, D. and Cheng, L. (2014) Fast linearized alternating direction minimization algorithm with adaptive parameter selection for multiplicative noise removal. Journal of Computational and Applied Mathematics, 257, 29-45.

2. Chernyi, S. (2016a) Use of Information Intelligent Components for the Analysis of Complex Processes of Marine Energy Systems,Transport and Telecommunication, 17(3), 202-211. DOI: $10.1515 / \mathrm{ttj}-2016-0018$

3. Chernyi, S. (2016b) Techniques for selecting topology and implementing the distributed control system network. IOP Conf. Ser.: Mater. Sci. Eng., 124, 012048.

4. Chernyi, S. (2016c) Analysis of the energy reliability component for offshore drilling platforms within the Black Sea. Neftyanoe Khozyaystvo - Oil Industry, 2, 106-110.

5. Cutroneo, L., Castellano, M., Carbone, C., Consani, S., Gaino, F., Tucci, S., Magrì, S., Povero, P., Bertolotto, R., Canepa, G. and Capello, M. (2015) Evaluation of the boundary condition influence on $\mathrm{PAH}$ concentrations in the water column during the sediment dredging of a port. Marine Pollution Bulletin, 101(2), 583-593.

6. Khoshgoftaar, T. and Seliya, N. (2003) Software Quality Classification Modelling Using the SPRINT Decision Tree Algorithm. International Journal on Artificial Intelligence Tools, 12(03), 207-225.

7. Malioutov, D., Corum, A. and Cetin, M. (2016) Covariance Matrix Estimation for Interest-Rate Risk Modeling via Smooth and Monotone Regularization. IEEE J. Sel. Top. Signal Process., 10(6), 10061014.

8. Nyrkov, A., Sokolov, S., Belousov, A. (2015) Algorithmic Support of Optimization of Multicast Data Transmission in Networks with Dynamic Routing. Modern Applied Science, 9(5), 162-76. DOI: 10.5539/mas.v9n5p162.

9. Park, C. (2005) Parameter estimation of incomplete data in competing risks using the EM algorithm. IEEE Transactions on Reliability, 54(2), 282-290. 
10. Rood, B. and Lewis, M. (2009) Grid Resource Availability Prediction-Based Scheduling and Task Replication. J Grid Computing, 7(4), 479-500.

11. Ruffio, E., Saury, D. and Petit, D. (2012) Robust experiment design for the estimation of thermo physical parameters using stochastic algorithms. International Journal of Heat and Mass Transfer, 55(11-12), 2901-2915.

12. Russell, M. (2014) Strategic scoping report and dredging effects. Marine Pollution Bulletin, 86(1-2), 594.

13. Shi, Y. and Ji, H. (2014) Smooth approximation method for non-smooth empirical risk minimization based distance metric learning. Neurocomputing, 127, pp.135-143.

14. Spearman, J. (2015) A review of the physical impacts of sediment dispersion from aggregate dredging. Marine Pollution Bulletin, 94(1-2), 260-277.

15. Tuan, Salwani Awang (2013) Enhancing Students' Understanding in Integral Calculus through the Integration of Maple in Learning. Procedia -Social and Behavioural Sciences, 102(22), 204-211.

16. Upadhya, K., Seelamantula, C. and Hari, K. (2016) A risk minimization framework for channel estimation in OFDM systems. Signal Processing, 128, 78-87.

17. Vieira, G., Neto, F. and Ribeiro, J. (2015) The Rationalization of Port Logistics Activities: A Study at Port of Santos (Brazil). International Journal of e-Navigation and Maritime Economy, 2, pp.73-86.

18. Walther, L., Rizvanolli, A., Wendebourg, M. and Jahn, C. (2016) Modelling and Optimization Algorithms in Ship Weather Routing. International Journal of e-Navigation and Maritime Economy, 4, 31-45. 